\title{
Case Report: Mucosal Leishmaniasis in New York City
}

\author{
Henry W. Murray, ${ }^{1 *}$ Daniel P. Eiras, ${ }^{1}$ Laura A. Kirkman, ${ }^{1}$ Raymond L. Chai, ${ }^{2}$ and Daniel Caplivski ${ }^{3}$ \\ ${ }^{1}$ Division of Infectious Diseases, Department of Medicine, Weill Cornell Medical College, New York, New York; ${ }^{2}$ Division of Infectious Diseases, \\ Department of Otolaryngology, Icahn School of Medicine at Mount Sinai, New York, New York; ${ }^{3}$ Division of Infectious Diseases, Department of \\ Medicine, Icahn School of Medicine at Mount Sinai, New York, New York
}

\begin{abstract}
The six previously reported civilian cases of mucosal leishmaniasis (ML) diagnosed in the United States have all represented imported New World ML. We describe two new patients with ML diagnosed in New York City-a Syrian immigrant with a nasal mass (Leishmania tropica), the first report of Old World ML in the United States, and an American ecologist who worked in Bolivia and had been treated for cutaneous infection 23 years before developing lesions (L. (Viannia) braziliensis) initially of the uvula, soft palate, and posterior pharynx and subsequently the larynx.
\end{abstract}

\section{INTRODUCTION}

Mucosal leishmaniasis (ML), one form of sand fly bitetransmitted protozoal infection, is seldom encountered in the United States. In civilians, only six cases of this uncommon, potentially destructive form of leishmaniasis appear to have been reported in U.S. citizens who have traveled to or worked in or in immigrants from regions where leishmaniasis, typically cutaneous leishmaniasis (CL), is endemic. ${ }^{1-6}$ In addition, at least three cases of ML diagnosed in this country have also been described in U.S. soldiers. ${ }^{7,8}$

Two of the six civilian cases were reported from New York City (NYC) - one in $2000^{2}$ and the other in $1975 .{ }^{1}$ Here, we describe two additional patients with $\mathrm{ML}$ cared for in the past several years at separate NYC teaching hospitals-an immigrant from Syria and a U.S. citizen who had worked in Bolivia.

\section{CASE REPORTS}

Patient 1. This patient was a 77-year-old Syrian man who had lived in NYC for 50 years and had not returned to Syria. Other than brief, once-a-decade trips to urban Israel, most recently, 6 years before presentation, he had not traveled to a leishmaniasis-endemic region. He had no recollection of prior cutaneous lesions nor treatment for leishmaniasis. In 2014, 3 months of nasal stuffiness and a sense of swelling led to otolaryngology evaluation, which revealed a large lobulated mass (Figure 1) originating from the septum and obstructing the entire left nasal airway. There were no oropharyngeal or laryngeal lesions. Computed tomography scan indicated some bowing of the nasal septum and edema without erosion. Biopsy of the mass showed granulomatous and mononuclear cell inflammation and numerous intracellular amastigotes (Figure 2). Polymerase chain reaction (PCR) testing of the tissue, performed at the New York State Department of Health, indicated infection caused by Leishmania tropica, and he was referred to the New York-Presbyterian Hospital/Weill Cornell Medical College for treatment. He was HIV negative, had no history of or had not undergone treatment to suggest underlying immunocompromise, and had no old cutaneous scars. Repeat nasoendoscopy (Figure 1) confirmed the presence of the left septal mass.

\footnotetext{
*Address correspondence to Henry W. Murray, Division of Infectious Diseases, Department of Medicine, Weill Cornell Medical College, 1300 York Ave., New York, NY 10065. E-mail: hwmurray@med. cornell.edu
}

His age and chronic renal (serum creatinine, $1.5 \mathrm{mg} / \mathrm{dL}$ ) and cardiac disease (atrial fibrillation and coronary artery disease) raised concern for adverse reactions to sodium stibogluconate or liposomal amphotericin B. Thus, oral miltefosine was given for 4 weeks at $150 \mathrm{mg} /$ day-the Food and Drug Administration-approved dose for individuals weighing $\geq$ $45 \mathrm{~kg}$ (he weighed $68 \mathrm{~kg}$ and, therefore, received $2.2 \mathrm{mg} / \mathrm{kg} /$ day $^{9}$ ). Treatment, given in three divided doses, was well tolerated, and his nasal symptoms responded within 2 weeks. One week after the therapy ended, repeat evaluation showed an $\sim 50 \%$ decrease in the size of the mass with reduced nasal obstruction. Over the next 2 months, his symptoms resolved nearly entirely, and 9 weeks after treatment, repeat otolaryngologic examination showed only a slightly raised "remnant" of infection. The patient elected not to return for followup; however, 6 months after treatment, he was asymptomatic according to his family. Efforts to recontact the patient and his family 4 years after treatment were unsuccessful.

Patient 2. This patient was a 45-year-old U.S. citizen who in 1995 (23 years before presentation) studied wildlife ecology in the Amazon rainforest of Bolivia. There, he developed a right neck skin lesion along with enlarged cervical lymph nodes. On return to the United States, skin lesion biopsy confirmed leishmaniasis, and after intravenous sodium stibogluconate treatment $(20 \mathrm{mg} / \mathrm{kg} /$ day for 21 days) and complete healing of the skin lesion, he returned to Bolivia for further work. He subsequently developed new skin lesions at other sites on his neck, also diagnosed as leishmaniasis. Before returning home, to stay, he was treated for 21 days in Bolivia with oncedaily intramuscular injections of meglumine antimoniate.

Years later in the United States, he developed a sore throat and difficult, painful swallowing that persisted for 6 months. Subsequent evaluation in late 2018 showed a deformed, ulcerated uvula with "cobblestoning" and ulceration on the soft palate and posterior pharynx, prompting admission to the Otolaryngology Service at the Icahn School of Medicine at Mount Sinai and resection of the uvula (Figure 3). There were, in particular, no nasal or laryngeal lesions on endoscopic sinonasal evaluation and flexible laryngoscopy, and he was HIV negative. Uvula tissue showed chronic inflammation with caseating granulomas and intracellular amastigotes; tissue culture and analysis performed at the CDC identified $L$. (Viannia) braziliensis.

The patient was treated with miltefosine, $150 \mathrm{mg} /$ day (in three divided doses), representing $\sim 2 \mathrm{mg} / \mathrm{kg} / \mathrm{day}$ at his body weight of $77 \mathrm{~kg}$. To spare him a fourth daily dose of $50 \mathrm{mg}$ (to 

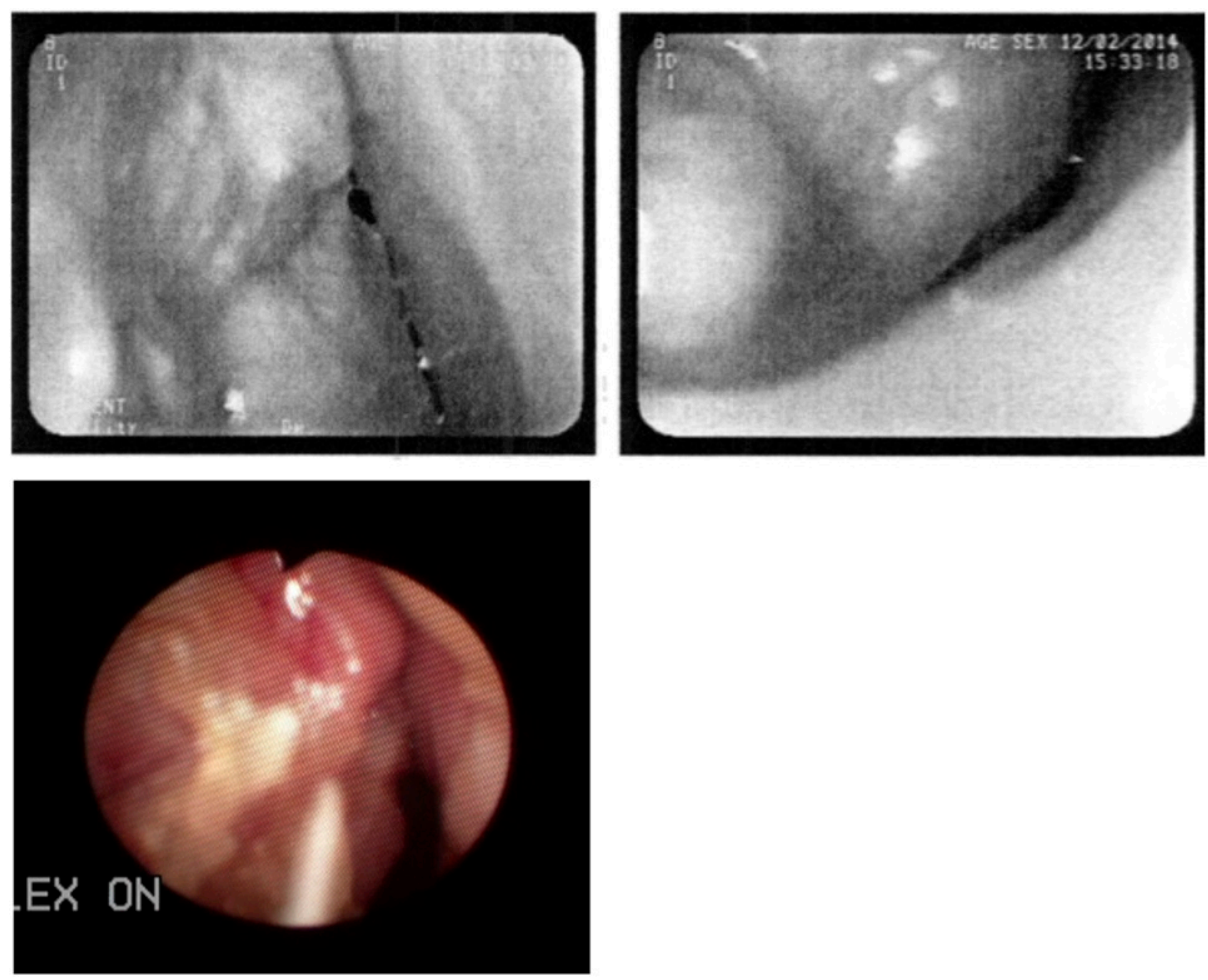

FIGURE 1. Patient 1. Pretreatment endoscopic views of lobulated left nasal mass obtained before (top) and after referral to Weill Cornell Medical College (bottom, left). This figure appears in color at www.ajtmh.org.

achieve a dose of $\sim 2.5 \mathrm{mg} / \mathrm{kg} /$ day as recommended in 2016$)^{9}$ and possible additional gastrointestinal reactions, thrice-daily treatment was extended from 4 to 8 weeks. Other than minor nausea, he tolerated the treatment well and had full resolution of his throat pain and swallowing difficulties. Four months after treatment, the resection site was healed, and the posterior pharyngeal ulcerations had resolved. However, 11 months after treatment, irritation on swallowing developed, prompting repeat endoscopy. Although the soft palate, posterior phar$\mathrm{ynx}$, and nasal mucosa were normal in appearance, flexible laryngoscopy showed new edema of the right aryepiglottic fold with cobblestoning of the laryngeal ventricle (Figure 3). Tissue biopsy suggested the presence of amastigotes, and $L$. (Viannia) braziliensis was again identified at the CDC. Retreatment is planned using liposomal amphotericin B (3 mg/kg/day, total dose $30 \mathrm{mg} / \mathrm{kg}^{9}$ ).

\section{DISCUSSION}

These two patients serve as a reminder that ML may indeed be encountered in the United States, albeit rarely, in U.S. citizens who have traveled to or worked in and immigrants from regions where leishmaniasis is endemic. Including our two patients, only eight cases of imported ML have now been described in published reports in civilians in the United States. ${ }^{1-6}$ However, recent case reports from Israel, ${ }^{10}$ the United Kingdom, ${ }^{11,12}$ and Austria ${ }^{13}$ have highlighted $\mathrm{ML}$ in travelers and long-term residents returning from South America (especially from Bolivia ${ }^{10}$ ) and from the Mediterranean region. ${ }^{11,12}$ In addition, at its multiple international sites which include the United States, the GeoSentinel Surveillance Network recently reported 40 cases of $\mathrm{ML}$ in returning travelers and migrants over a 20 -year period. ${ }^{14}$ In that analysis, ML primarily occurred in tourists returning from South or Central America, nearly half of whom had been in Bolivia, and when performed $(n=14)$, speciation identified $L$. (V.) braziliensis in all cases. ${ }^{14}$

\section{PREVIOUSLY REPORTED CASES OF ML DIAGNOSED IN THE UNITED STATES}

New World ML. Similar to Patient 2, 1) infection in the six previously reported cases was acquired originally in South or Central America (Brazil: 2, Peru: 1, Bolivia: 1, El Salvador: 1, and unknown: 1); 2) four of the six were immigrants, but at least one case was also in a traveler (a zoologist) who worked in Peru; and 3) when performed, speciation demonstrated $L$. $(V$.$) braziliensis in three of four cases (and L. p$ in one). ${ }^{1-6}$ Among at least four New World species capable of involving the nasal, oropharyngeal, and/or laryngeal mucosa, L. (V.) braziliensis has been best studied and is particularly well recognized for involving the nasal mucosa (in $>90 \%$ of cases). ${ }^{15}$ Isolated involvement of the uvula and surrounding soft palate or subsequently the larynx alone, as in Patient 2, is unusual. ${ }^{15}$ Similar to Patient 2, the majority of those with New World ML have evidence of prior or concomitant cutaneous infection; however, as many as $20 \%$ do not, ${ }^{15}$ raising the possibility of inapparent skin infection caused by parasite strains with particular tropism for mucosal sites. In addition and like Patient 2's experience, having received prior pentavalent antimony treatment for $C L$ does not necessarily prevent the subsequent development of ML. For example, in a study of Brazilian patients with $M L$ caused by $L$. (V.) braziliensis, ${ }^{15}$ nearly one-third of those with prior CL had previously received standard antimony therapy for cutaneous infection. Finally, as in Patient 2, it is well recognized that 

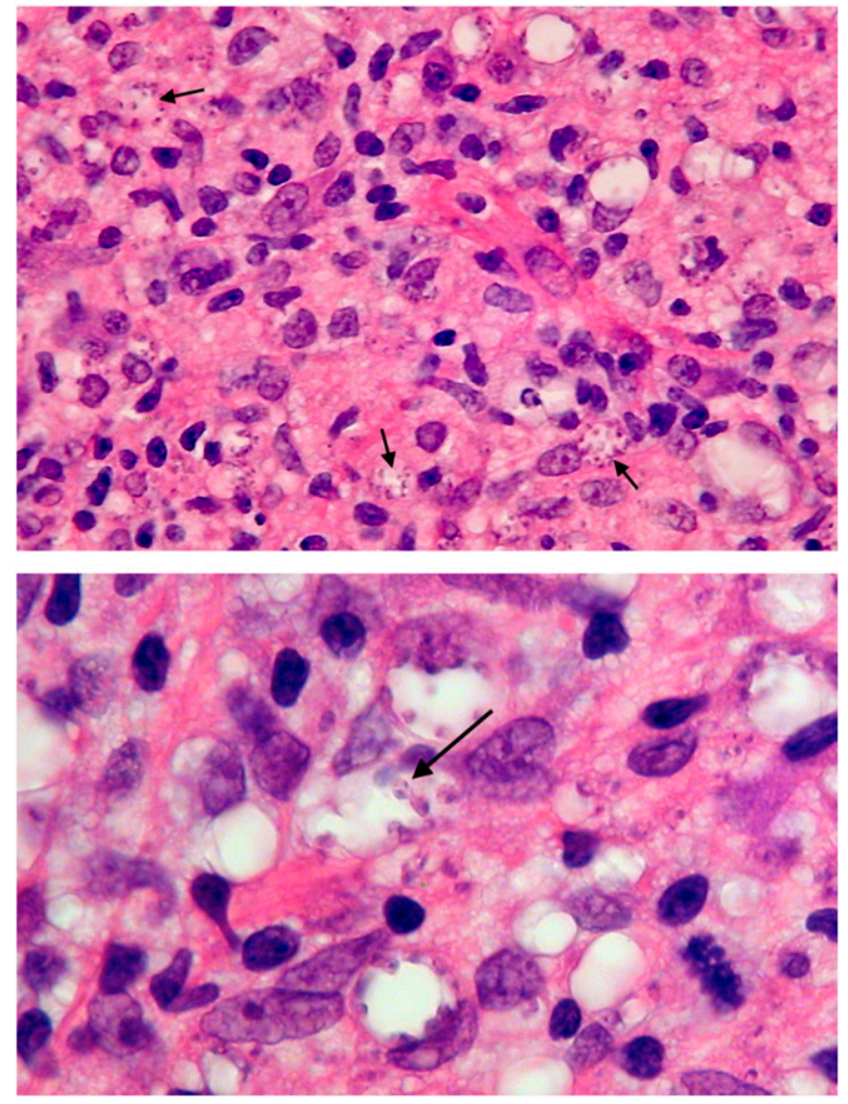

FIGURE 2. Photomicrographs of hematoxylin and eosin-stained tissue sections from biopsy of nasal mass. Top: Note mononuclear cell inflammatory infiltrate and numerous intracellular amastigotes, particularly within enlarged macrophage phagocytic vacuoles (arrows). Bottom: High-power view showing intracellular amastigotes within phagocytic vacuoles; arrow indicates an amastigote showing characteristic oval shape and darkly stained round nucleus and rodshaped kinetoplast. Original magnifications: $\times 400$ (top) and $\times 630$ (bottom). This figure appears in color at www.ajtmh.org.

years may elapse between detection of $C L$ and initial expression of ML. ${ }^{10,15}$

Overall, estimates suggest that $\mathrm{ML}$ complicates $<5 \%$ of cases of New World (American) tegumentary leishmaniasis, although the frequency of ML in patients with CL appears to be higher in Bolivia. ${ }^{10,16}$ As in Patient 2, and in addition to acquisition of infection in a high Andean country (most notably Bolivia), postulated risk factors for developing $\mathrm{ML}$ in patients with $\mathrm{CL}$ include having multiple $(>4)$ or large $(>4 \mathrm{~cm})$ or persistent lesions ( $>4$ months), localization of the skin lesion(s) on the head or neck or above the waist, inadequate prior treatment, and immunosuppression. ${ }^{10,16}$

Old World ML. Patient 1, infected with L. tropica, represents the first case of Old World ML reported in this country. Old World leishmaniasis, caused by infection with Leishmania donovani complex (L. donovani and Leishmania infantum) or Leishmania major complex (L. major and L. tropica), can on occasion be complicated by $\mathrm{ML}$ as well as imported into non-endemic countries. $^{13,17,18}$ Such reports reemphasize that mucosal infection is not confined to South America ${ }^{11}$ and that infection with any of the four species listed earlier can produce ML. In the GeoSentinel Network analysis of ML in travelers and migrants, four of the 40 patients acquired infection in the Old World (North Africa: 2 and Western Europe: 2). ${ }^{14}$ Mucosal areas other than the nose are more frequently affected in Old World ML (unlike Patient 1), and ML associated with $L$. infantum infection appears best studied. ${ }^{11,17}$

It is possible that Patient 1 originally acquired $L$. tropica infection in Syria, a highly endemic country, and that mucosal infection remained quiescent for at least 50 years before his presentation in NYC. It is also possible that during one of his subsequent trips to urban Israel, he acquired inapparent cutaneous infection which localized hematogenously to or even experienced direct infection ${ }^{18}$ of his nasal mucosa. Why infection subsequently reactivated and then progressed is not clear. He had no apparent immunocompromising disorder, had not been a smoker, had no upper respiratory tract infections, and had not used inhaled corticosteroids, which are apparent risk factors for developing Old World ML. ${ }^{11,16}$

\section{DIAGNOSIS}

There was no reason to suspect ML in Patient 1. By contrast, ML was considered in Patient 2 given his history of prior CL acquired in Bolivia, albeit more than two decades before and previously treated. In both patients, the initial diagnosis of ML was readily confirmed by conventional tissue histopathology which showed characteristic intracellular amastigotes (Figure 2);

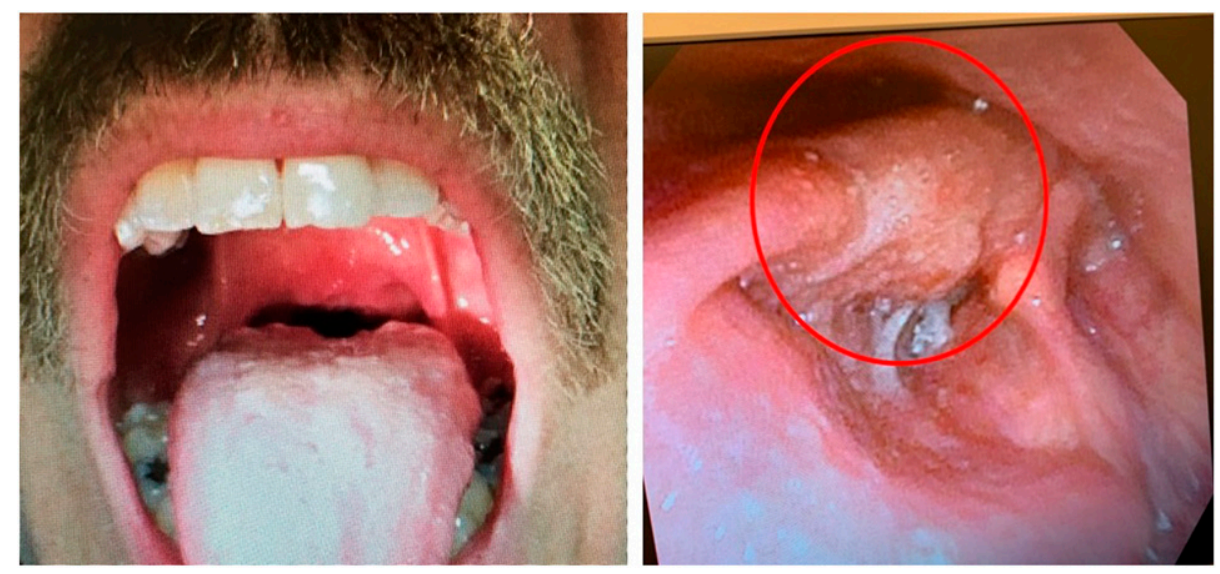

FigurE 3. Patient 2. Left: Postoperative photograph showing absent uvula and ulcerated and cobblestone appearance of soft palate and posterior pharyngeal wall. Preoperatively (photographs not performed), the uvula showed similar changes. Right: Laryngoscopic view 11 months after initial treatment showing edematous right aryepiglottic fold and mucosal cobblestoning (circled) of the laryngeal ventricle. This figure appears in color at www.ajtmh.org. 
speciation was achieved by culture and molecular analysis (DNA testing by PCR) at a reference laboratory. Although perhaps not the case in Old World ML, ${ }^{11,17}$ it is worth noting that despite typical histologic evidence of marked mononuclear cell and/or granulomatous inflammation, mucosal amastigotes may be scant and, thus, difficult to visualize microscopically in biopsied or resected tissue. Thus, if ML is suspected, multiple diagnostic methods should always be used in all patients. ${ }^{9}$ In addition, in the appropriate setting - an immigrant or returned traveler, worker, or expatriate from a leishmaniasis-endemic region who has a histologically demonstrated, midline-type of inflammatory granulomatous disease-ML should be considered and first investigated before empiric immunosuppressive therapy is begun. In three of the six previous reported U.S. cases, ${ }^{1,4,6} \mathrm{ML}$ presented in just such a fashion, and two were given likely deleterious immunosuppressive agents for extended periods before proper diagnosis and antileishmanial therapy.

\section{TREATMENT}

We treated our patients with oral miltefosine to which Patient 1 appeared to respond well clinically, although follow-up was incomplete. Patient 2 responded with resolution of his pharyngeal disease, but 11 months after the therapy developed new laryngeal lesions requiring retreatment. No treatment of choice has been identified for either New or Old World ML, and advice to individualize treatment has been suggested.9 However, heretofore traditional options for ML (pentavalent antimony and amphotericin B deoxycholate) have little appeal because both represent arduous parenteral therapies. By contrast, and although data are limited and/or uncontrolled, both intravenous liposomal amphotericin B and oral miltefosine are considered viable alternatives. ${ }^{9}$ Miltefosine has been used in both New World ${ }^{10,19-21}$ and Old World ML $^{11,22}$ with apparent satisfactory effects. However, as with all forms of leishmaniasis, including advanced $\mathrm{ML}$, and irrespective of the treatment selected, initial responses and long-term cures may be difficult to achieve. In addition, retreatment may be required and recurrence after apparent clinical cure is always a possibilityPatient 2 is a clear-cut example of this clinical problem. In patients with ML who show improvement or healing after treatment, most relapses occur within the first 2 years, ${ }^{9}$ thus the importance of close clinical follow-up.

Received November 20, 2019. Accepted for publication February 26, 2020.

Published online March 30, 2020.

Authors' addresses: Henry W. Murray and Laura A. Kirkman, Division of Infectious Diseases, Department of Medicine, Weill Cornell Medical College, New York, NY, E-mails: hwmurray@med.cornell.edu and hwmurray@med.cornell.edu. Daniel P. Eiras, New York City Department of Health and Mental Hygiene, New York, NY, E-mail: deiras@health.nyc.govRaymond L. Chai, Division of Head and Neck Surgical Oncology, Icahn School of Medicine at Mount Sinai, New York, NY, E-mail: raymond.chai@mountsinai.org. Daniel Caplivski, Division of Infectious Diseases, Icahn School of Medicine at Mount Sinai, New York, NY, E-mail: daniel.caplivski@mountsinai.org.

\section{REFERENCES}

1. Singer C, Armstrong D, Jones TC, Spiro RH, 1975. Imported mucocutaneous leishmaniasis in New York city. Report of a patient treated with amphotericin B. Am J Med 59: 444-447.
2. Huna-Baron R, Warren FA, Miller W, Jacobs J, Green J, Kupersmith MJ, 2000. Mucosal leishmaniasis presenting as sinusitis and optic neuropathy. Arch Ophthalmol 118: 852-854.

3. Costa JW, Milner DA, Maguire JH, 2003. Mucocutaneous leishmaniasis in a US citizen. Oral Surg Oral Med Oral Pathol Oral Radiol Endod 96: 573-577.

4. Brahn E, Pegues DA, Craft N, 2010. Mucocutaneous leishmaniasis as Wegener's granulomatosis. J Clin Rheumatol 16: 125-128.

5. Stein DJ, Yarlagadda BB, Noordzij JP, 2013. Otolaryngologic presentation of mucosal leishmaniasis. J Case Rep Med 1: 1-2.

6. Tejura N, Kim E, Dever L, Chew D, 2019. Case report: mucocutaneous leishmaniasis masquerading as idiopathic midline granulomatous disease. Am J Trop Med Hyg 101: 1107-1110.

7. Walton BC, Person DA, Bernstein R, 1968. Leishmaniasis in the U.S. Military on the canal zone. Am J Trop Med Hyg 17: 19-24.

8. Wortmann G, Zapor M, Ressner R, Fraser S, Hartzell J, Pierson J, Weintrob A, Magill A, 2010. Liposomal amphotericin B for treatment of cutaneous leishmaniasis. Am J Trop Med Hyg 83: 1028-1033.

9. Aronson $\mathrm{N}$ et al., 2016. Diagnosis and treatment of leishmaniasis: clinical practice guidelines by the Infectious Diseases Society of America (IDSA) and the American Society of Tropical Medicine and Hygiene (ASTMH). Clin Infect Dis 63: 1539-1557.

10. Solomon M, Sahar N, Paviotzky F, Barzilai A, Jaffe CL, Nasereddin A, Schwartz E, 2019. Mucosal leishmaniasis in travelers with Leishmania braziliensis complex returning to Israel. Emerg Infect Dis 25: 642-648.

11. Patel TA, Scadding GK, Phillips DE, Lockwood DN, 2017. Case report: old world mucosal leishmaniasis: report of five imported cases to the hospital for tropical diseases, London, United Kingdom. Am J Trop Med Hyg 97: 1116-1119.

12. Santangeli L, McCluney NA, Hathorn I, Shakeel M, Anderson C, 2009. Leishmaniasis presenting to the otolaryngologist: a rare but important cause of persistent hoarseness. J Laryngol Otology 123: 1181-1183.

13. Harrison N, Walochnik J, Ramsebner R, Veletzky L, Lagler $\mathrm{H}$, Rambarter M, 2017. Case report: progressive perforation of the nasal septum due to Leishmania major: a case of mucosal leishmaniasis in a traveler. Am J Trop Med Hyg 96: 653-655.

14. Boggild AK et al., 2019. Cutaneous and mucocutaneous leishmaniasis in travellers and migrants: a 20-year GeoSentinel surveillance network analysis. J Travel Med 26: taz055.

15. Cincura C, de Lima CMF, Machado PRL, Oloveira-Filho J, Glesby MJ, Lessa M, Carvalho EM, 2017. Mucosal leishmaniasis: a retrospective study of 327 cases from an endemic area of 15 . Leishmania (Viannia) braziliensis. Am J Trop Med Hyg 97: 761-766.

16. Blum $\mathrm{J}$ et al., 2014. LeishMan recommendations for treatment of cutaneous and mucosal leishmaniasis in travelers, 2014. J Travel Med 21: 116-129.

17. Aliaga L, Cobo F, Mediavilla JD, Bravo J, Osuna A, Aamador JM, Martin-Sanchez J, Cordero E, Navarro JM, 2003. Localized mucosal leishmaniasis due to Leishmania (Leishmania) infantum: clinical and microbiologic findings in 31 patients. Medicine (Baltimore) 82: 147-158.

18. Strazzulla A, Cocuzza S, Pinzone MR, Postorino MC, Cosentino S, Serra A, Cacopardo B, Nunnari G, 2013. Mucosal leishmaniasis: an underestimated presentation of a neglected disease. Biomed Res Int 2013: 203108.

19. Soto $\mathrm{J}$ et al., 2007. Treatment of Bolivian mucosal leishmaniasis with miltefosine. Clin Infect Dis 44: 350-356.

20. Soto J, Rea J, Valderrama M, Toledo J, Valda L, Ardiles J, Berman J, 2009. Efficacy of extended (six weeks) treatment with miltefosine for mucosal leishmaniasis in Bolivia. Am J Trop Med Hyg 81: 387-389.

21. Ventin F, Cincura C, Machado PRL, 2018. Safety and efficacy of miltefosine monotherapy and pentoxifylline associated with pentavalent antimony in treating mucosal leishmaniasis. Expert Rev Anti Infect Ther 16: 219-225.

22. Mosimann V, Blazek C, Gron H, Chaney M, Neumayr A, Blum J, 2016. Miltefosine for mucosal and complicated cutaneous old world leishmaniasis: a case series and review of the literature. Open Forum Infect Dis 3: ofw008. 